\title{
On Robe's Circular Restricted Problem of Three Variable Mass Bodies
}

\author{
Jagadish Singh ${ }^{1}$ and Oni Leke ${ }^{2}$ \\ ${ }^{1}$ Department of Mathematics, Faculty of Science, Ahmadu Bello University Zaria, PMB 2222, Samaru-Zaria, Kaduna, Nigeria \\ ${ }^{2}$ Department of Mathematics, College of Science, University of Agriculture, PMB 2373, North-Bank, Makurdi, Nigeria
}

Correspondence should be addressed to Oni Leke; lekkyonix4ree@yahoo.com

Received 25 March 2013; Accepted 25 August 2013

Academic Editors: M. Biesiada, K. Bolejko, M. S. Dimitrijevic, M. Jamil, and E. Saridakis

Copyright (C) 2013 J. Singh and O. Leke. This is an open access article distributed under the Creative Commons Attribution License, which permits unrestricted use, distribution, and reproduction in any medium, provided the original work is properly cited.

\begin{abstract}
This paper investigates the motion of a test particle around the equilibrium points under the setup of the Robe's circular restricted three-body problem in which the masses of the three bodies vary arbitrarily with time at the same rate. The first primary is assumed to be a fluid in the shape of a sphere whose density also varies with time. The nonautonomous equations are derived and transformed to the autonomized form. Two collinear equilibrium points exist, with one positioned at the center of the fluid while the other exists for the mass ratio and density parameter provided the density parameter assumes value greater than one. Further, circular equilibrium points exist and pairs of out-of-plane equilibrium points forming triangles with the centers of the primaries are found. The out-of-plane points depend on the arbitrary constant $\kappa$, of the motion of the primaries, density ratio, and mass parameter. The linear stability of the equilibrium points is studied and it is seen that the circular and out-of-plane equilibrium points are unstable while the collinear equilibrium points are stable under some conditions. A numerical example regarding out-of-plane points is given in the case of the Earth, Moon, and submarine system. This study may be useful in the investigations of dynamic problem of the "ocean planets" Kepler-62e and Kepler-62f orbiting the star Kepler-62.
\end{abstract}

\section{Introduction}

The classical restricted three-body problem (RTBP) constitutes one of the most important problems in dynamical astronomy. The study of this problem is of great theoretical, practical, historical, and educational relevance. The investigation of this problem in its several versions has been the focus of continuous and intense research activity for more than two hundred years. The study of this problem in its many variants has had important implications in several scientific fields including, among others, celestial mechanics, galactic dynamics, chaos theory, and molecular physics. The RTBP is still a stimulating and active research field that has been receiving considerable attention of scientists and astronomers because of its applications in dynamics of the solar and stellar systems, lunar theory, and artificial satellites.

A different kind of restricted three-body problem was formulated by Robe [1], a set up in which the first primary is a rigid spherical shell filled with homogenous, incompressible fluid of density $\rho_{1}$, and the second primary is a mass point outside the shell and moving around the first primary in a Keplerian orbit, while the infinitesimal mass is a small sphere of density $\rho_{3}$ moving inside the shell and is subject to the attraction of the second primary and the buoyancy force due to the fluid.

In estimating buoyancy force, Robe [1] assumed that the pressure field of the fluid $\rho_{1}$ has spherical symmetry around the center of the shell, and he considered only one out of the three components of the pressure field, which is due to the own gravitational field of the fluid $\rho_{1}$.

A. R. Plastino and A. Plastino [2] took into account all these components of pressure field. But in their study, they assumed the hydrostatic equilibrium figure of the first primary as Roche's ellipsoid. They found that when the density parameter $D$ is zero, every point inside the fluid is an equilibrium point; otherwise, the ellipsoid's center is the only equilibrium point. They also examined the linear stability of equilibrium points. Hallan and Rana [3] investigated the existence of all equilibrium point and their stability in the Robe's [1] problem. It was seen that the Robe's elliptic restricted 
three-body problem has only one equilibrium point for all values of the density parameter and the mass parameter, while the Robe's circular restricted three-body problem can have two, three, or infinite numbers of equilibrium points. As regards to the stability of these equilibria, they found that the collinear equilibrium points are stable while triangular and circular points are always unstable. Recently, Kaur and Aggarwal [4] investigated the Robe's problem of $2+2$ bodies and applied it to the study of the motion of two submarines in the Earth-Moon system. Singh and Hafsah [5] examined the Robe's circular restricted three-body problem when the first primary is a fluid in the shape of an oblate spheroid and the second primary is a triaxial rigid body.

The classical restricted three-body problem assumes that the masses of celestial bodies are constant. However, the phenomenon of isotropic radiation or absorption in stars led scientists to formulate the restricted problem of three bodies with variable mass. As an example, we could mention the motion of rockets, black holes formation, motion of a satellite around a radiating star surrounded by a cloud and varying its mass due to particles of the cloud, and comets loosing part or all of their mass as a result of roaming around the Sun (or other stars) due to their interaction with the solar wind which blows off particles from their surfaces. The problem of the motion of astronomical objects with variable mass has many interesting applications in stellar, galactic, and planetary dynamics.

The study of two bodies with variable masses seems to have been first investigated by Dufour [6] where he examined the astronomical phenomena of variable mass relating the secular variation of lunar acceleration with the increase of the Earth's mass due to the impact of meteorites. Later, Gylden [7] wrote the differential equations of motion for the problem when the masses are subject to variation. The integrable case to this differential equation was then given by Meshcherskii $[8,9]$. The problem was later known as the GyldenMeshcherskii problem. A characterization of this problem was studied by Singh and Leke [10]. The effect of the isotropic variation of the mass of the star in a planetary system and the possible ejection of a planet from the system were studied by Veras et al. [11]. Recently, Singh and Leke [12] investigated the existence and stability of equilibrium points in the Robe's restricted three-body problem with variable masses.

Besides the Gylden-Meshcherskii problem, there are other different cases of two bodies with variable masses, which are classified according to the presence or absence of reactive forces, to whether the bodies move in an inertial frame or not, and so on (see [13]). For instance, when the particles are at rest in an inertial coordinate system, this case may be used to study the orbits of a celestial body moving through a static atmosphere, whose particles attach to it or detach from it as it moves. The restricted three-body problem with nonisotropic variation of the masses has been studied by Bekov [14], Bekov et al. [15], and Letelier and Da Silva [16]. A simple example of this kind of problem is the system of two variable primaries and a rocket. In this case, it is the thrust from the rocket that defines the force that acts on the test particle, in addition to the gravitational attraction from the two primaries, while the rocket does not affect the orbits of the primaries.

In this paper, the existence and stability of equilibrium points under the frame of the Robe problem [1], when the participating bodies vary their masses at the same rate, is studied. Here, we assume that the primaries move in a stationary medium, from which they absorb or lose mass; the first primary being a fluid in the shape of a sphere and the test particle which is a small sphere located inside the fluid also gain or lose mass to the fluid. Hence, there is no need to assume a rigid spherical shell. This study may be useful in the investigations of dynamic the problem of water-planetary system discovered by Kepler spacecraft. These "ocean planets" are orbiting the star Kepler-62 and are designated Kepler62e and Kepler-62f. The existence of these Earth-size planets covered completely by a water envelope (water planets) has long fascinated scientists and the general public. The model of this problem can also be used to study the small oscillation of the Earth's inner core taking into account the Moon's attraction during the course of evolution.

This paper is orginzed as follows: Section 2 contains the equations of motion; the equilibrium points are investigated in Section 3; Section 4 investigates the linear stability of the equilibrium points; Section 5 discusses the obtained results and the conclusions.

\section{Equations of Motion}

Let $m_{1}$ be the mass of the first primary which is a fluid in the shape of a sphere of radius $\mathfrak{R}$ with center at $M_{1}$ having density $\delta_{1}$ and volume $V_{1}$. Also, let $m_{2}$ be the mass of the second primary with center at $M_{2}$ which describes a circular orbit around the first one. Both masses are assumed to vary with time as they travel in a static medium which acts as a sink or source of mass. Now, let $m_{3}$ be the mass of the test particle whose mass is very small compared with the masses of the primaries, with center at $M_{3}$, having density $\delta_{3}$ and volume $V_{3}$. We suppose that its mass varies with time, also as it moves about in the fluid, it gains or loses mass to the medium. Let the positions vector between the center of the fluid and the centers of the second and the test particle be $\vec{r}_{12}$ and $\vec{r}_{13}$, respectively, and let that between the test particle and the second primary be $\vec{r}_{23}$. Following Robe [1] and knowing that the masses, distances, and densities vary with time, the forces acting on the third body are the force of attraction of $m_{2}$; the gravitational force $\vec{F}_{A}$ exerted by the fluid, that is $\vec{F}_{A}=$ $-\left(4 \Re^{3} / 3 r_{13}^{3}\right) \pi G \delta_{1} m_{3} \overrightarrow{M_{1} M_{3}}$; and the buoyancy force exerted by the fluid which is $\vec{F}_{B}=\left(4 \pi \Re^{3} / 3 r_{13}\right) G \delta_{1}^{2}\left(m_{3} / \delta_{3}\right) \overrightarrow{M_{3} M_{1}}$.

We adopt a rotating coordinate system $O x y z$ with origin at the center of mass, $O$, of the primaries, $O x$ pointing towards the second primary, and $O x y$ being the orbital plane of $m_{2}$. The equations of motion of the test particle, taking into account the forces acting on it, have the following form $[1,14]$ :

$$
\begin{aligned}
\ddot{x}-2 \omega \dot{y}= & \omega^{2} x+\dot{\omega} y-\frac{4 \pi \Re^{3}\left(x-x_{1}\right)}{3 r_{13}^{3}} \\
& \times G \delta_{1}\left(1-\frac{\delta_{1}}{\delta_{3}}\right)-\frac{\mu_{2}\left(x-x_{2}\right)}{r_{23}^{3}}-\frac{\dot{m}_{3}}{m_{3}}(\dot{x}-\omega y),
\end{aligned}
$$




$$
\begin{aligned}
& \ddot{y}+2 \omega \dot{x}= \omega^{2} y-\dot{\omega} x-\frac{4 \pi \Re^{3} y}{3 r_{13}^{3}} \\
& \times G \delta_{1}\left(1-\frac{\delta_{1}}{\delta_{3}}\right)-\frac{\mu_{2} y}{r_{23}^{3}}-\frac{\dot{m}_{3}}{m_{3}}(\dot{y}-\omega x), \\
& \ddot{z}=\frac{4 \pi \Re^{3} z}{3 r_{13}^{3}} G \delta_{1}\left(1-\frac{\delta_{1}}{\delta_{3}}\right)-\frac{\mu_{2} z}{r_{23}^{3}}-\frac{\dot{m}_{3} \dot{z}}{m_{3}},
\end{aligned}
$$

where $r_{13}^{2}=\left(x-x_{1}\right)^{2}+y^{2}+z^{2}, r_{23}^{2}=\left(x-x_{2}\right)^{2}+y^{2}+z^{2}$.

The barycentric coordinates $x_{1}$ and $x_{2}$ are connected with the distance between the primaries by the following equations:

$$
\mu_{1}(t)=\mu \frac{x_{2}}{r_{12}}, \quad \mu_{2}(t)=-\mu \frac{x_{1}}{r_{12}},
$$

where $\mu_{1}(t)=G m_{1}(t), \mu_{2}(t)=G m_{2}(t), m_{1}(t)=\delta_{1} V_{1} ; V_{1}=$ $4 \pi \Re^{3} / 3$, while $G$ is the gravitational constant and the over dot denotes differentiation with respect to time $t$.

Now, in order to obtain useful dynamical predictions, we transform $(x, y, z, t)$ to the autonomous form $(\xi, \eta, \zeta, \tau)$. Following [15], the time dependence of the masses is described [15] by the function $\mu(t)$ :

$$
\begin{aligned}
& x=\left(\frac{\mu_{0}}{\mu}\right)^{3} \xi, \quad y=\left(\frac{\mu_{0}}{\mu}\right)^{3} \eta, \\
& z=\left(\frac{\mu_{0}}{\mu}\right)^{3} \zeta, \quad \frac{d t}{d \tau}=\left(\frac{\mu_{0}}{\mu}\right)^{5}, \\
& \omega(t)=\omega_{0}\left(\frac{\mu}{\mu_{0}}\right)^{5}, \quad \mu_{i}=\mu_{0 i} \frac{\mu}{\mu_{0}}, \\
& m_{3}=m_{03} \frac{\mu}{\mu_{0}}, \quad r_{i 3}=\rho_{i 3}\left(\frac{\mu_{0}}{\mu}\right)^{3}, \\
& r_{12}=\rho_{12}\left(\frac{\mu_{0}}{\mu}\right)^{3}, \quad(i=1,2) .
\end{aligned}
$$

As in [14], the particular solutions for the case with variable parameters $\mu_{1}, \mu_{2}$, and $m_{3}$ in the form of the Eddington-Jeans laws with indices $n=3$ and $n=6$ is expressed with the help of the function $\mu$ :

$$
\dot{\mu}=\alpha_{1} \mu_{1}^{n}, \quad \dot{\mu}=\alpha_{2} \mu_{2}^{n}, \quad \dot{m}_{3}=\alpha m_{3}^{n},
$$

where $\alpha, \alpha_{1}$, and $\alpha_{2}$ are constants. The exponent $n=1$ falls in the stellar range while $n=2$ and $n=3$ result, respectively, in the first and second law of Meshcherskii $[8,9]$ mass variations. Equations of (4) indicate that the laws of variation of the three masses are the same.

Also, the dynamical system has the particular solution of the following type $[15,17]$ :

$$
r_{12} \mu m_{3}^{2}=\kappa C^{2},
$$

where $\kappa>0$ is a constant, and $C \neq 0$ is a constant of the area integral.
Finally, in addition, we assume that the densities of the fluid and the test particle vary such that

$$
\delta_{1}(t)=\mu \frac{\delta_{01}}{\mu_{0}}, \quad \delta_{3}(t)=\mu \frac{\delta_{03}}{\mu_{0}},
$$

where $\delta_{01}$ and $\delta_{03}$ are the densities of the medium and the test particle, respectively, at initial time $t_{0}$.

Substituting (3) to (6) in (1) and reducing it throughout by $\mu^{7} / \mu_{0}^{7}$, we get

$$
\xi^{\prime \prime}-2 \omega_{0} \eta^{\prime}=\Omega_{\xi}, \quad \eta^{\prime \prime}+2 \omega_{0} \xi^{\prime}=\Omega_{\eta}, \quad \zeta^{\prime \prime}=\Omega_{\zeta},
$$

where

$$
\begin{aligned}
\Omega= & \frac{\kappa \omega_{0}^{2}\left(\xi^{2}+\eta^{2}+\zeta^{2}\right)}{2}-\frac{\omega_{0}^{2} \zeta^{2}}{2} \\
& +\frac{\mu_{02}}{\rho_{23}}-G \frac{D}{2}\left[\left(\xi-\xi_{1}\right)^{2}+\eta^{2}+\zeta^{2}\right], \\
\rho_{13}^{2}= & \left(\xi-\xi_{1}\right)^{2}+\eta^{2}+\zeta^{2}, \\
\rho_{23}^{2}= & \left(\xi-\xi_{2}\right)^{2}+\eta^{2}+\zeta^{2}, \\
\xi_{1}= & \frac{-\mu_{20}}{\mu_{0}} \rho_{12}, \quad 2 \xi_{2}=\frac{\mu_{10}}{\mu_{0}} \rho_{12}, \\
D= & \frac{4 \pi}{3} \delta_{01}\left(1-\frac{\delta_{01}}{\delta_{03}}\right) .
\end{aligned}
$$

Here, $\rho_{12}$ is constant and connects the parameter $\kappa$ by the relation

$$
\rho_{12} \mu_{0}=\kappa C^{2} .
$$

Equations (5) and (9) indicate that the ratio of the product of the distances between the center of the primaries, mass of the test particle and the sum of the masses with the gravitational constant to the constant of the area integral, always remains a constant in both the autonomous and the non autonomous systems.

Now, we choose units for the distance and time, such that at initial time $t_{0}, \rho_{12}=1, \omega_{0}=C=1$, respectively. Putting these in (9), for the unit of sum of the masses, we get $\mu_{0}=$ $G=\kappa$.

Next, without loss of generality, we introduce the mass parameter defined as $v=\mu_{20} / \mu_{0}(0<v<1)$ and also assume that the pressure field of the fluid of density $\delta_{01}$ maintains a spherical symmetry around the center of the fluid such that $\rho_{13}=\Re$. Also, we have $\xi_{1}=-v$ and $\xi_{2}=1-v$. With the help of these units, the system of (7) takes the following form:

$$
\xi^{\prime \prime}-2 \eta^{\prime}=\Omega_{\xi}, \quad \eta^{\prime \prime}+2 \xi^{\prime}=\Omega_{\eta}, \quad \zeta^{\prime \prime}=\Omega_{\zeta},
$$

where

$$
\begin{aligned}
\Omega(\xi, \eta, \zeta) & =\frac{\kappa\left(\xi^{2}+\eta^{2}\right)}{2}+\frac{(\kappa-1) \zeta^{2}}{2}+\frac{\kappa v}{\rho_{23}}-\frac{\kappa D}{2} \rho_{13}^{2}, \\
\rho_{13}^{2} & =(\xi+v)^{2}+\eta^{2}+\zeta^{2}, \\
\rho_{23}^{2} & =(\xi+v-1)^{2}+\eta^{2}+\zeta^{2},
\end{aligned}
$$


and the dash signifies differentiation with respect to the new time $\tau$.

Equations (10) are the autonomized equations of motion of the test particle of our problem. These equations are different from that in [18] and analogous to the equations in Hallan and Rana [3] only differing due to the second term that appears in the force function and the parameter $\kappa$.

\section{The Equilibrium Points}

The equilibrium points represent stationary solutions of the RTBP. These solutions are the singularities of the manifold of the components of the velocity and the coordinates and are found by setting $\xi^{\prime}=\eta^{\prime}=\zeta^{\prime}=\xi^{\prime \prime}=\eta^{\prime \prime}=\zeta^{\prime \prime}=0$ in the equations of motion (10). That is, they are the solutions of the equations $\Omega_{\xi}=\Omega_{\eta}=\Omega_{\zeta}=0$, which are

$$
\begin{aligned}
& \xi-\frac{v(\xi+v-1)}{\rho_{23}^{3}}-D(\xi+v)=0, \\
& \left(1-\frac{v}{\rho_{23}^{3}}-D\right)=0, \quad \eta \neq 0, \\
& \left(\frac{\kappa-1}{\kappa}-\frac{v}{\rho_{23}^{3}}-D\right)=0, \quad \zeta \neq 0 .
\end{aligned}
$$

The solutions are categorized as follows.

(1) The solutions of first equation of (12) with $\eta=\zeta=0$ yield the collinear equilibrium points. These points lie on the line joining the center of the first and second primary.

(2) When the first and the second equation of (12) are solved with $\zeta=0$, we get the circular points. These points lie in the spherical fluid and form circles.

(3) The solutions of first and the third equations of (12) with $\eta=0$ results in the out-of-plane equilibrium points. These solutions are valid provided they lie inside the fluid. We shall consider them in Sections 3.1, 3.2, and 3.3, respectively.

3.1. Collinear Points. From first equation of (12), with $\eta=\zeta=$ 0 , we get

$$
\begin{gathered}
(\xi+v)\left[(1-D) \xi^{2}+(v-2+2 D-2 D v) \xi\right. \\
\left.+1+2 D v-D v^{2}-D\right]=0
\end{gathered}
$$

Hence, (13) has three roots, the first being $\xi=-v$ and is always a solution whether $D=0$ or is not, for $0<\kappa<\infty$. The two remaining roots are found by considering the second equality in (13), which gives

$$
\xi_{2,3}=\frac{-2+v+2 D(1-v) \pm \sqrt{\Delta}}{2(D-1)},
$$

where $\Delta=v(4 D+v-4)$.
Now, the solutions exist only for $D>1$, however, the second solution is greater than $1-v$ and consequently will lie outside the shell, so we ignore it. The third solution is

$$
\xi_{3}=\frac{-2+v+2 D(1-v)-\sqrt{\Delta}}{2(D-1)}
$$

and is less than $1-v$, the $\xi$-coordinate of the second primary. Hence, there are two collinear equilibrium points which lie on the line joining the centers of the primaries. Therefore, the coordinate $(-v, 0,0)$ is always an equilibrium point. For $D>$ 1 , there exist an equilibrium point $\left(\xi_{3}, 0,0\right)$ which lies to the left or right of the first primary depending upon whether $D<$ $1+2 v$ or $D>1+2 v$. When $D=1+2 v$, the only equilibrium point is the center of the shell.

3.2. Circular Points. These solutions are found by solving the first and second equations of (12) with $\zeta=0$. Solving the second equation of (12) gives

$$
D=1-\frac{v}{\rho_{23}^{3}}
$$

Substituting (16) in the first equation of (12) yields $D=1-v$ and consequently $\rho_{23}^{3}=1$.

Hence, we have the solution

$$
(\xi+v-1)^{2}+\eta^{2}=1, \quad D=1-v,
$$

which gives the coordinate of any point on the circle (17) with center $(1-v, 0,0)$ which is the center of the second primary and radius one which is the distance between the centers of the fluid and the second primary. Thus, the solution gives us an infinite number of equilibrium points, provided they lie inside the fluid.

3.3. Out-of-Plane Points. The out-of-plane equilibrium points are found by solving first and third equations of (12) with $\eta=$ 0 . Solving first for $\rho_{23}$ in the third equation of (12), we get

$$
\rho_{23}=\left(\frac{\kappa v}{\kappa-1-\kappa D}\right)^{1 / 3} .
$$

Substituting (18) in the first equation of (12) and simplifying results in

$$
\xi=(\kappa-1)(v-1)+\kappa D
$$

Knowing that $\rho_{23}^{3}=(\xi+v-1)^{2}+\zeta^{2}$, substituting (18) and (19) in it, and solving for $\zeta$, we get

$$
\zeta= \pm \sqrt{\left(\frac{\kappa v}{\kappa-1-\kappa D}\right)^{2 / 3}-\kappa^{2}(1-v-D)^{2}} .
$$

Equations (19) and (20) give the position $(\xi, 0, \zeta)$ of real outof-plane equilibrium points provided $\kappa \geq 1 /(1-D)$. Should $\kappa<1 /(1-D)$, then no real out-of-plane points exist as (20) turns out to be imaginary or complex quantity. When $\kappa=1$, these points fully coincide with that of Hallan and Rana [3]. 


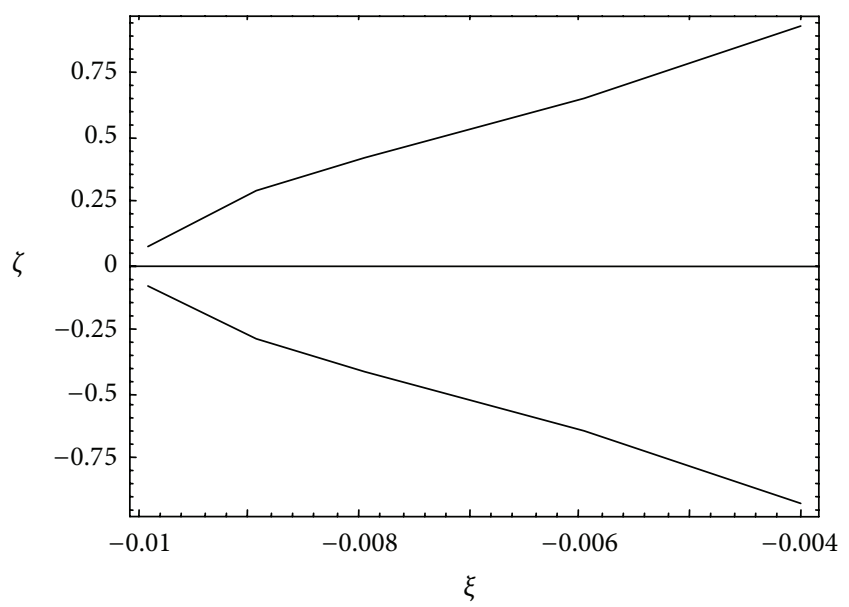

FIGURE 1: Out-of-plane points for, $v=0.01$ and $D=-0.001$.

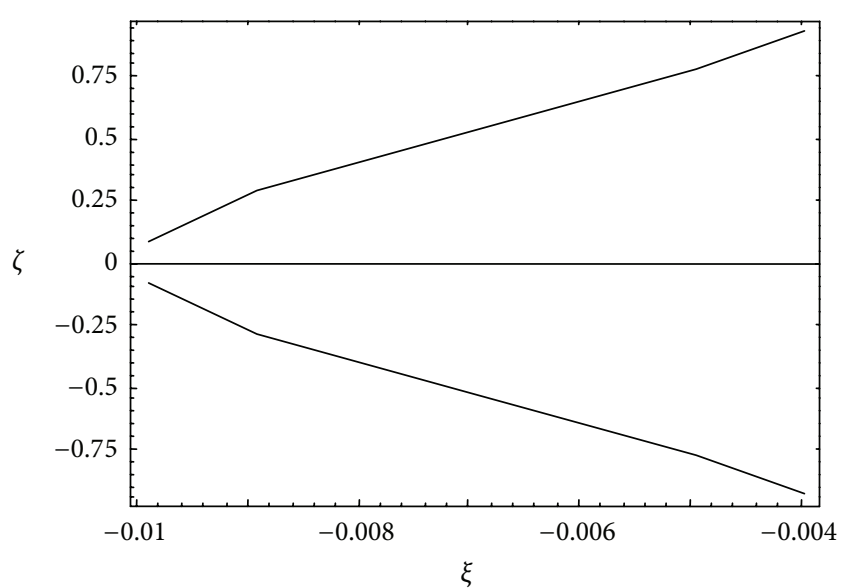

Figure 2: Out-of-plane points for, $v=0.01$ and $D=0$.

When $D=0$, (i.e., $\delta_{01}=\delta_{03}$ ), the coordinates of the out-ofplane points become the same with that in Singh and Leke [12]. Hence, it is seen that the equilibrium points are fully analogous to those found by Hallan and Rana [3] except for the out-of-plane equilibrium points which are affected by the parameter $\kappa$, the density parameter, and the mass ratio. The positions of the out-of-plane points are given in Tables 1, 2 and 3 and their graphical representations in Figures 1, 2, and 3 for a test particle in the Earth-Moon system, when the density parameter $D$ is negative, zero, and positive, respectively.

We summarize our numerical effort as follows. In Table 1 , when $D=-0.001$ this implies that $\delta_{03}<\delta_{01}$. In this case for $0<\kappa \leq 0.999$ and $1.009<\kappa<\infty$, out-of-plane points do not exist, but however exist in the interval $0.999001 \leq \kappa \leq$ 1.999. In Table $2, D=0$ and so $\delta_{03}=\delta_{01}$. In this case the out-of-plane points exist only when $1.001 \leq \kappa \leq 1.01$ and do not exist in the remaining entire range of $\kappa$, while for Table 3 , $D=0.001$ and so $\delta_{03}>\delta_{01}$. Here, real out-of-plane solutions exist, for the values of the parameter $\kappa$, in the interval $1.002 \leq$ $\kappa \leq 1.0111$ and are nonexistent for any value of $\kappa$ outside this range. Hence, though $\kappa$ has a large range of values, however, the physically meaningful range is $\kappa \in[0.999001,1.0111]$, at which the out-of-plane points exist (see Figure 4). However,

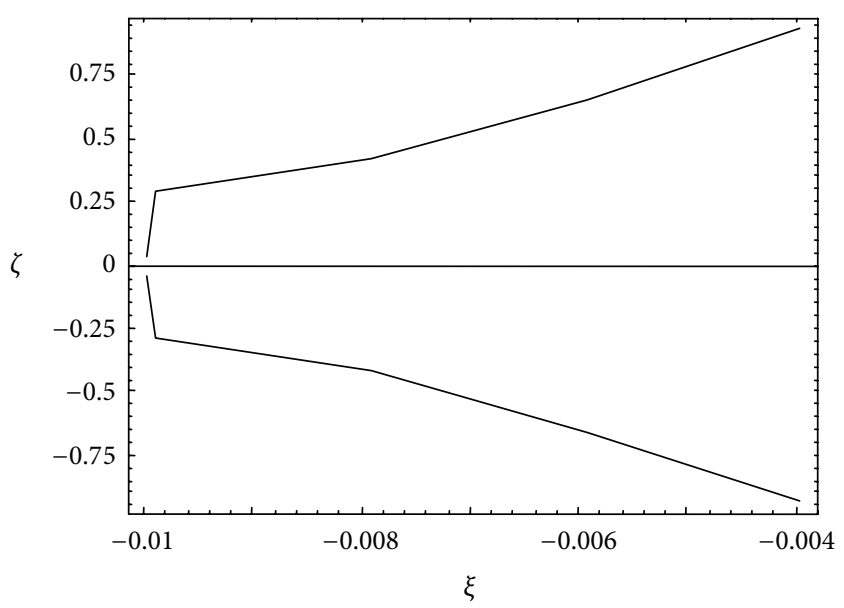

Figure 3: Out-of-plane points for $v=0.01$ and $D=0.001$.

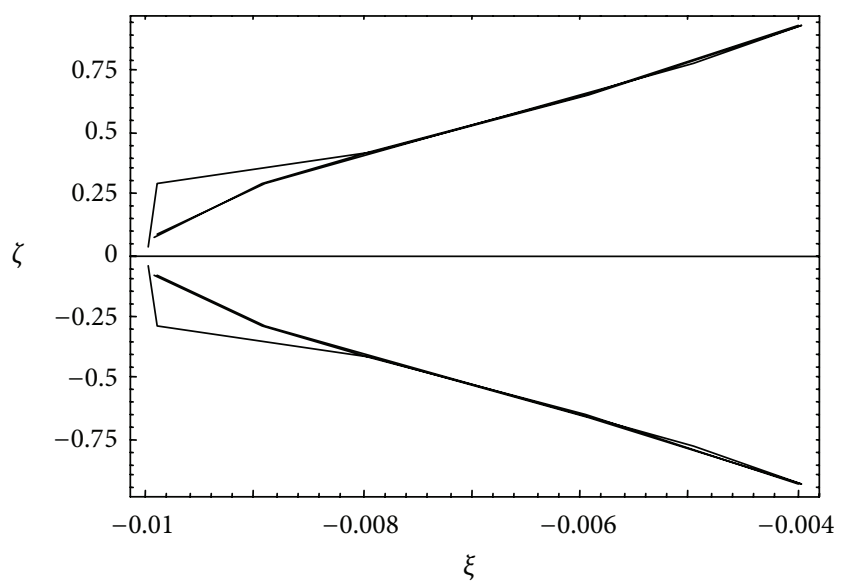

FIGURE 4: Out-of-plane points for, $v=0.01$ and $\kappa \in[0.999001$, $1.0111]$.

these ranges may differ for different density parameters $D$, which are determined by the densities of the fluid and the test particle.

\section{Stability of Equilibrium Points}

To examine the stability of an equilibrium configuration, that is, its ability to restrain the body motion in its vicinity, we apply small displacement $u, v, w$ to the coordinates $\left(\xi_{0}, \eta_{0}, \zeta_{0}\right)$ of the third body, to the positions, $\xi=\xi_{0}+u, \eta=\eta_{0}+v$ and $\zeta=\zeta_{0}+w$. If its motion rapidly departs from the vicinity of the point, we call such a position of equilibrium an unstable one. If however the body merely oscillates about the point, it is said to be a stable position.

Now, we linearize (10) to obtain the variational equations:

$$
\begin{aligned}
u^{\prime \prime}-2 v^{\prime} & =\left(\Omega_{\xi \xi}^{0}\right) u+\left(\Omega_{\xi \eta}^{0}\right) v+\left(\Omega_{\xi \zeta}^{0}\right) w, \\
v^{\prime \prime}+2 u^{\prime} & =\left(\Omega_{\xi \eta}^{0}\right) u+\left(\Omega_{\eta \eta}^{0}\right) v+\left(\Omega_{\eta \zeta}^{0}\right) w, \\
w^{\prime \prime} & =\left(\Omega_{\xi \zeta}^{0}\right) u+\left(\Omega_{\eta \zeta}^{0}\right) v+\left(\Omega_{\zeta \zeta}^{0}\right) w,
\end{aligned}
$$


TABLE 1: The out-of-plane points for $v=0.01$ and $D=-0.001$.

\begin{tabular}{lccc}
\hline$\kappa$ & $\xi$ & $\pm \zeta$ & Comments \\
\hline 0.01 & 0.98009 & $0.0232859+0.0403325 i$ & Real out of plane point do not exist \\
0.5 & 0.4945 & Complex & - \\
0.999 & Real and negative & Complex & Real out-of-plane points exist \\
0.999001 & - & 215.369 & - \\
1 & -0.001 & 1.91758 & - \\
1.001 & -0.011 & 1.3988 & - \\
1.002 & -0.002982 & 1.12303 & Real out-of-plane point exist \\
1.003 & -0.003973 & 0.932023 & - \\
1.004 & -0.004964 & 0.781521 & - \\
1.005 & -0.005955 & 0.652458 & - \\
1.006 & -0.006946 & 0.533794 & - \\
1.007 & -0.007937 & 0.416357 & - \\
1.008 & -0.008928 & 0.28657 & - \\
1.009 & -0.009919 & 0.0755768 & Real out-of-plane point do not exist \\
$1.009<\kappa<\infty$ & Real and negative & Imaginary &
\end{tabular}

TABLE 2: The out-of-plane points for $v=0.01$ and $D=0$.

\begin{tabular}{|c|c|c|c|}
\hline$\kappa$ & $\xi$ & $\pm \zeta$ & Comments \\
\hline 0.01 & 0.9801 & Complex & Real out of plane point do not exist \\
\hline 0.5 & 0.9801 & - & - \\
\hline 0.999 & 0.00099 & - & - \\
\hline 0.999001 & 0.00098901 & - & - \\
\hline 1 & 0 & Infinity & Infinite remote solution \\
\hline 1.001 & -0.00099 & 1.9138 & Real out-of-plane points exist \\
\hline 1.002 & -0.00198 & 1.39986 & - \\
\hline 1.003 & -0.00297 & 1.12416 & - \\
\hline 1.004 & -0.00396 & 0.933202 & - \\
\hline 1.005 & -0.00495 & 0.782774 & - \\
\hline 1.006 & -0.00594 & 0.653819 & - \\
\hline 1.007 & -0.00693 & 0.535323 & - \\
\hline 1.008 & -0.00792 & 0.418177 & - \\
\hline 1.009 & -0.00891 & 0.289043 & - \\
\hline 1.0091 & -0.009009 & 0.274411 & - \\
\hline 1.01 & -0.0099 & 0.0839973 & - \\
\hline $1.01<\kappa<\infty$ & Real and negative & Imaginary & Real out-of-plane point do not exist \\
\hline
\end{tabular}

where the superscript 0 indicates that the partial derivatives are to be evaluated at the equilibrium points.

4.1. Collinear Points. Robe [1] discussed the stability of the equilibrium point at the center of the shell, and Hallan and Rana [3] have also discussed that in the case of the noncollinear points when $D<0$. Hence, we shall discuss here the stability of the equilibrium point $\left(\xi_{3}, 0,0\right)$ near the center of the fluid. To do this, we let solutions of the first two equations of (21) be $u=A \exp (\lambda \tau), v=B \exp (\lambda \tau)$, where $A, B$, and $\lambda$ are constants.

Finding first and second derivatives of the solutions, substituting them in the first two equations of (21), and simplifying, we obtain the matrix which has a nonzero solution when

$$
\left|\begin{array}{ll}
\left(\lambda^{2}-\Omega_{\xi \xi}^{0}\right) & \left(2 \lambda+\Omega_{\xi_{\eta}}^{0}\right) \\
\left(2 \lambda-\Omega_{\xi_{\eta}}^{0}\right) & \left(\lambda^{2}-\Omega_{\eta \eta}^{0}\right)
\end{array}\right|=0 .
$$

Expanding the determinant, we get

$$
\lambda^{4}-\left(\Omega_{\xi \xi}^{0}+\Omega_{\eta \eta}^{0}-4\right) \lambda^{2}+\Omega_{\xi \xi}^{0} \Omega_{\eta \eta}^{0}-\left(\Omega_{\xi \eta}^{0}\right)^{2}=0 .
$$

This is the characteristic equation corresponding to the variational equations (21) when motion is considered in the $\xi \eta$-plane. 
TABLE 3: The out-of-plane points for $v=0.01$ and $D=0.001$.

\begin{tabular}{|c|c|c|c|}
\hline$\kappa$ & $\xi$ & $\pm \zeta$ & Comments \\
\hline 0.01 & 0.98011 & Complex & Real out-of-plane point do not exist \\
\hline 0.5 & 0.4955 & - & - \\
\hline 0.999 & 0.001989 & - & - \\
\hline 0.999001 & 0.00198801 & - & - \\
\hline 1 & 0.001 & - & - \\
\hline 1.001 & 0.000011 & - & - \\
\hline 1.002 & -0.000978 & 1.92083 & Real out-of-plane point exist \\
\hline 1.003 & -0.001967 & 1.40162 & - \\
\hline 1.004 & -0.002956 & 1.12573 & - \\
\hline 1.005 & -0.003945 & 0.934716 & - \\
\hline 1.006 & -0.004934 & 0.784303 & - \\
\hline 1.007 & -0.005923 & 0.655425 & - \\
\hline 1.008 & -0.006912 & 0.537083 & - \\
\hline 1.009 & -0.007901 & 0.420232 & - \\
\hline 1.0091 & -0.0079999 & 0.408219 & - \\
\hline 1.01 & -0.0099 & 0.291786 & - \\
\hline 1.0111 & -0.0099779 & 0.0393474 & - \\
\hline $1.0112<\kappa<\infty$ & Real and negative & Complex & Real out-of-plane point do not exist \\
\hline
\end{tabular}

Now, the values of the second order partial derivatives computed at the point $\left(\xi_{3}, 0,0\right)$, with the substitution $\eta=\zeta=$ 0 are as follow:

$$
\begin{aligned}
& \Omega_{\xi \xi}^{0}=\kappa\left(1-D+4 A_{1}\right), \\
& \Omega_{\eta \eta}^{0}=\kappa\left(1-D-2 A_{1}\right), \\
& \Omega_{\xi \zeta}^{0}=\Omega_{\eta \zeta}^{0}=\Omega_{\xi \eta}^{0}=0, \\
& \Omega_{\zeta \zeta}^{0}=\kappa\left(\frac{\kappa-1}{\kappa}-2 A_{1}-D\right),
\end{aligned}
$$

where $A_{1}=\left(4 v(D-1)^{3}\right) /[v+\sqrt{v(4 D+v-4)}]^{3}$.

When $\kappa=1$, the equations in system (24) fully coincide with those of Hallan and Rana [3].

Substituting (24) in the variational equations (21), at once gives

$$
\begin{aligned}
& u^{\prime \prime}-2 v^{\prime}=\kappa\left(1-D+4 A_{1}\right) u, \\
& v^{\prime \prime}+2 u^{\prime}=\kappa\left(1-D-2 A_{1}\right) v, \\
& w^{\prime \prime}=\kappa\left(\frac{\kappa-1}{\kappa}-2 A_{1}-D\right) w .
\end{aligned}
$$

Now, (26) is independent of (25) and depicts that the motion parallel to the $\zeta$-axis is stable provided $1 \leq \kappa<1 /\left(1-2 A_{1}-\right.$ $D)$.

Now, the characteristic equation (23) using (24) becomes

$$
\begin{aligned}
\lambda^{4} & +\lambda^{2}\left\{2 \kappa\left(\frac{2}{\kappa}-1+D-A_{1}\right)\right\} \\
& +\kappa^{2}\left(D-1-4 A_{1}\right)\left(D-1+2 A_{1}\right)=0,
\end{aligned}
$$

where $D>1, A_{1}>0$.
The roots of (28) are

$$
\Lambda_{n}=\frac{-P \pm \sqrt{\Delta}}{2}, \quad(n=1,2),
$$

where

$$
\begin{aligned}
& P=2 \kappa\left(\frac{2}{\kappa}-1+D-A_{1}\right), \\
& \Delta=4\left\{4 \kappa\left(D-A_{1}\right)-4(\kappa-1)+9 \kappa^{2} A_{1}^{2}\right\},
\end{aligned}
$$

$\Delta$ is the discriminant of (27) and is always positive for any $\kappa$. When $\kappa=1$, the value of $\Delta$ fully coincides with that of Hallan and Rana [3] which ought to be $\Delta=4\left\{4\left(D-A_{1}\right)+9 A_{1}^{2}\right\}$.

Now, since $\Delta>0$, if in (28) the quantity in parenthesis is positive; that is, $P>0$, which occurs when

$$
0<\kappa<\frac{2}{1+A_{1}-D}
$$

we see that $P>\sqrt{\Delta}$. Hence from (28), we have $-P \pm \sqrt{\Delta}<$ 0 , and so both values of $\Lambda_{n}$ are negative and consequently, the roots (28) are distinct pure imaginary. Therefore, the equilibrium point $\left(\xi_{3}, 0,0\right)$ is stable provided equation (30) holds; otherwise, it is unstable.

4.2. Circular Points. These equilibrium points exist only for $D=1-v$. The coordinates of any point on the circle $(\xi+v-1)^{2}+\eta^{2}=1, \zeta=0$ are of the form $(1-v-$ $\cos \phi, \sin \phi, 0)$. 
The partial derivatives at these points are

$$
\begin{aligned}
& \Omega_{\xi \xi}^{0}=3 \kappa v \cos ^{2} \phi, \\
& \Omega_{\eta \eta}^{0}=3 \kappa v \sin ^{2} \phi, \\
& \Omega_{\zeta \zeta}^{0}=-1, \\
& \Omega_{\xi \eta}^{0}=-3 \kappa v \sin \phi \cos \phi, \\
& \Omega_{\eta \zeta}^{0}=\Omega_{\xi \zeta}^{0}=0 .
\end{aligned}
$$

When these are substituted in the variational equations (21) with $\zeta=0$, we have

$$
\begin{aligned}
u^{\prime \prime}-2 v^{\prime} & =3 \kappa v \cos \phi(u \cos \phi-v \sin \phi), \\
v^{\prime \prime}+2 u^{\prime} & =-3 \kappa v \sin \phi(u \cos \phi-v \sin \phi), \\
w^{\prime \prime} & =-w .
\end{aligned}
$$

The last equation of (32) shows that motion is stable along the $\zeta$-axis. The characteristic equation of the first two equations of (32) is

$$
\lambda^{2}\left(\lambda^{2}-3 \kappa v+4\right)=0
$$

Its roots are $\lambda_{1,2}=0, \lambda_{3,4}= \pm \sqrt{-4+3 \kappa v}$, and so the equilibrium points are unstable due to multiple zero roots.

4.3. Out-of-Plane Points. For the stability of the out-of-plane equilibrium points, we consider the following partial derivatives:

$$
\begin{aligned}
& \Omega_{\xi \xi}^{0}=1+\frac{3 \kappa^{2}(1-v-D)^{2}(\kappa-1-\kappa D)^{5 / 3}}{(\kappa v)^{2 / 3}}, \\
& \Omega_{\eta \eta}^{0}=1, \\
& \Omega_{\xi \eta}^{0}=\Omega_{\eta \zeta}^{0}=0, \\
& \Omega_{\xi \zeta}^{0}=\frac{-3 \zeta \kappa(1-v-D)(\kappa-1-\kappa D)^{5 / 3}}{(\kappa v)^{2 / 3}}, \\
& \Omega_{\zeta \zeta}^{0}=\frac{3 \zeta^{2}(\kappa-1-\kappa D)^{5 / 3}}{(\kappa v)^{2 / 3}} .
\end{aligned}
$$

The characteristic equation in this case is gotten by substituting the trial solutions $u=A \exp (\lambda \tau), v=B \exp (\lambda \tau)$, $w=C \exp (\lambda \tau)$ in the variational equations (21) to get

$$
\lambda^{6}+p \lambda^{4}+q \lambda^{2}+r=0
$$

where

$$
\begin{aligned}
p= & 4-\Omega_{\xi \xi}^{0}+\Omega_{\eta \eta}^{0}+\Omega_{\zeta \zeta}^{0}, \\
q= & \Omega_{\eta \eta}^{0}\left(\Omega_{\xi \xi}^{0}+\Omega_{\zeta \zeta}^{0}\right) \\
& +\Omega_{\zeta \zeta}^{0}\left[\Omega_{\xi \xi}^{0}-4\left(\Omega_{\xi \eta}^{0}\right)^{2}\right]-\left(\Omega_{\xi \zeta}^{0}\right)^{2}-\left(\Omega_{\eta \zeta}^{0}\right)^{2}, \\
r= & \Omega_{\eta \eta}^{0}\left(\Omega_{\xi \zeta}^{0}\right)^{2}+\left(\Omega_{\eta \zeta}^{0}\right)\left[\Omega_{\eta \zeta}^{0} \Omega_{\xi \xi}^{0}-2 \Omega_{\xi \eta}^{0} \Omega_{\xi \zeta}^{0}\right] \\
& +\Omega_{\zeta \zeta}^{0}\left[\left(\Omega_{\xi \eta}^{0}\right)^{2}-\Omega_{\xi \xi}^{0} \Omega_{\eta \eta}^{0}\right] .
\end{aligned}
$$

Substituting (34) in (35), we at once have

$$
\begin{aligned}
\lambda^{6}+[5 & -3 \kappa(1-D)] \lambda^{4} \\
+ & {\left[1-\frac{3(\kappa-1-\kappa D)}{v^{2 / 3}}\right.} \\
& \left.\times\left\{2 v^{2 / 3}-3 \kappa^{4 / 3}(1-v-D)^{2}(\kappa-1-\kappa D)^{2 / 3}\right\}\right] \lambda^{2} \\
+ & 3(1+\kappa D-\kappa) \\
& \times\left[1-\frac{\kappa^{4 / 3}(\kappa-1-\kappa D)(1-v-D)^{2}}{v^{2 / 3}}\right]=0 .
\end{aligned}
$$

Its roots are

$$
\begin{aligned}
\lambda_{1,2} \longrightarrow \pm & {\left[\frac{p}{3}+\frac{\left(p^{2}-q\right)}{3}\left(\frac{2}{N}\right)^{1 / 3}+\frac{N^{1 / 3}}{32^{1 / 3}}\right]^{1 / 2}, } \\
\lambda_{3,4} \longrightarrow \pm & {\left[\frac{p}{3}-\frac{p^{2}}{32^{2 / 3} N^{1 / 3}}+\frac{i p^{2}}{2^{1 / 3} \sqrt{3} N^{1 / 3}}+\frac{q}{2^{2 / 3} N^{1 / 3}}\right.} \\
& \left.-\frac{i \sqrt{3 q}}{2^{2 / 3} N^{1 / 3}}+\frac{N^{1 / 3}}{22^{1 / 3} \sqrt{3}}+\frac{N^{1 / 3}}{62^{1 / 3}}\right]^{1 / 2}, \\
\lambda_{5,6} \pm & {\left[\frac{p}{3}-\frac{p^{2}}{32^{2 / 3} N^{1 / 3}}-\frac{i p^{2}}{2^{2 / 3} \sqrt{3} N^{1 / 3}}+\frac{q}{2^{2 / 3} N^{1 / 3}}\right.} \\
& \left.+\frac{i \sqrt{3 q}}{2^{2 / 3} N^{1 / 3}}+\frac{N^{1 / 3}}{22^{1 / 3} \sqrt{3}}-\frac{N^{1 / 3}}{62^{1 / 3}}\right]^{1 / 2},
\end{aligned}
$$

where $N=2 p^{3}-9 p q+27 r+$ $\sqrt{4\left(3 q-p^{2}\right)^{3}+\left(2 p^{3}-9 p q+27 r\right)^{2}}, p=5-3 \kappa(1-D), q=1-$ $\left(3(\kappa-1-\kappa D) / v^{2 / 3}\right)\left\{2 v^{2 / 3}-3 \kappa^{4 / 3}(1-v-D)^{2}(\kappa-1-\kappa D)^{2 / 3}\right\}$, $r=3(1+\kappa D-\kappa)\left[1-\kappa^{4 / 3}(\kappa-1-\kappa D)(1-v-D)^{2} / v^{2 / 3}\right]$.

The roots (37) are computed numerically for motion of a test particle (a submarine) under the gravitational attraction of the Earth-Moon system when $D>0$ and $D<0 \quad\left(\delta_{01}<\delta_{03}\right.$ and $\delta_{01}>\delta_{03}$, resp.) for $0<\kappa<\infty$. Therefore, we take $v=$ 0.01 and $\kappa \in[0.999001,1.0111]$ with the following values in each case following Kaur and Aggarwal [4]. 
TABLE 4: The characteristic roots $\lambda_{1,2}= \pm \omega_{1}, \lambda_{3,4}= \pm \omega_{2}, \lambda_{5,6}= \pm \omega_{3}$ for $v=0.01, D=-2572.699$.

\begin{tabular}{lccc}
\hline$\kappa$ & $\omega_{1}$ & $\omega_{2}$ & $\omega_{3}$ \\
\hline 0.999001 & $3526.63+3526.09 i$ & $2.13681 i$ & $3526.63-3526.09 i$ \\
1 & $3529.28+3528.73 i$ & $2.13716 i$ & $3529.28-3528.73 i$ \\
1.001 & $3531.93+3531.38 i$ & $2.13752 i$ & $3531.93-3531.38 i$ \\
1.002 & $3534.57+3534.03 i$ & $2.13787 i$ & $3534.57-3534.03 i$ \\
1.003 & $3537.22+3536.67 i$ & $2.13823 i$ & $3537.22-3536.67 i$ \\
1.004 & $3539.86+3539.32 i$ & $2.13859 i$ & $3539.86-3539.32 i$ \\
1.009 & $3553.08+3552.53 i$ & $2.14036 i$ & $3553.08-3552.53 i$ \\
1.01 & $3555.72+3555.17 i$ & $2.14071 i$ & $3555.72-3555.17 i$ \\
1.0111 & $3558.62+3558.08 i$ & $2.1411 i$ & $3558.62-3558.08 i$ \\
\hline
\end{tabular}

In the case when $\delta_{01}<\delta_{03}$, we take mass of test particle $m_{3}=1848632 \mathrm{~kg}$, density of salt water $\delta_{01}=1027 \mathrm{~kg} / \mathrm{m}^{3}$, density of test particle $\delta_{03}=1204.39 \mathrm{~kg} / \mathrm{m}^{3}$.

In new units, we have

$$
\delta_{01}=9317.7, \delta_{03}=10927.11 \text {, and so } D=1372.4 \text {. }
$$

Similarly, when $\delta_{01}>\delta_{03}$, we take

$$
\begin{aligned}
& \text { mass of test particle } m_{3}=1449832 \mathrm{~kg}, \\
& \text { density of salt water } \delta_{01}=1027 \mathrm{~kg} / \mathrm{m}^{3}, \\
& \text { density of submarine } \delta_{03}=804.79 \mathrm{~kg} / \mathrm{m}^{3} .
\end{aligned}
$$

In new units, we have

$$
\delta_{01}=9317.7, \delta_{03}=7301.65 \text {, and so } D=-2572.699 \text {. }
$$

Aside from these examples, we also consider the case when $D= \pm 0.001$ (see Tables 1 and 3), so that a wider generalization can be reached regarding the characteristic roots (38) which consequently determines whether the equilibrium point is a stable one or not.

Using the software package Mathematica; the six characteristic roots are presented in Tables 4, 5, 6, and 7 numerically for $v=0.01$, for different density parameters and a wide range of the parameter $\kappa$. We seek to find the case where all the six roots are pure imaginary quantities or complex figures with negative real parts. If this happens, then the solutions will be bounded and motion will be stable; otherwise, they will be unstable.

From these tables, we see that that for a specific set of values of these parameters at least one of the roots among all has a positive real part or a complex root with the existence of a positive real part. Therefore, this causes the solutions to be unbounded and consequently producing unstable equilibrium points. Hence, we conclude that the out-of-plane equilibrium points are unstable equilibrium points due to a positive root and positive real part in complex roots. This agrees with the result of Singh [5].

The equilibrium solutions of the nonautonomous system with variable coefficients are in general unstable points according to the Lyapunov's theorem of stable solutions [19].

\section{Discussion and Conclusion}

We have derived the equations of motion and established the possible equilibrium points of the third body of infinitesimal mass in a setup of Robe's [1] restricted three-body problem when the three participating bodies all vary their masses arbitrarily at the same rate and the density of the fluid and third body also vary as the masses. We find that the nonautonomous equations of motion are different from that of the restricted problem of three variable mass bodies derived by Bekov et al. [15], while the autonomized equations of our study are also different from those of Robe [1] and Hallan and Rana [3] due to the assumptions we have introduced here. The autonomized system of (6) is different from that in Hallan and Rana [3] only due to an additional term which appears due to the parameter $\kappa$. When $\kappa=1$, the equations become fully analogous to theirs.

The equilibrium points are sought, and it is seen that the point at the center of the fluid is always an equilibrium point of the Robe problem. An equilibrium point near the center of the fluid, points on the circle (circular points), and two out-of-plane points on the $\xi \zeta$-plane also exist, with all similar to that found in Hallan and Rana [3]; though the later have several points in our case while there exists only a pair in that of Hallan and Rana [3].

The linear stability of the equilibrium points of the autonomized have been studied and the outcomes are analogous with the stability results in Hallan and Rana [3], in the sense that the equilibrium points collinear with the centers of the fluid and the second primary are stable under some given conditions which depend on the mass ratio, density parameter, and the parameter $\kappa$. The circular are unstable due to the presence of multiple zero roots, while the out-of-plane equilibrium points are also unstable due to a positive root and a positive real part of the complex roots.

In our recent paper, Singh and Leke [12], the motion of a test particle around the equilibrium points was generalized to include the effect of mass variations of the primaries which vary isotropically in accordance with the unified Meshcherskii law, when the motion of the primaries is determined by the Gylden-Meshcherskii problem. Here, ejection or attachment form or to the surfaces of the primaries do not create reactive forces. Also, we have taken the first primary as 
TABLE 5: The characteristic roots $\lambda_{1,2}= \pm \omega_{1}, \lambda_{3,4}= \pm \omega_{2}, \lambda_{5,6}= \pm \omega_{3}$ for $v=0.01$ and $D=-0.001$.

\begin{tabular}{lccc}
\hline$\kappa$ & $\omega_{1}$ & $\omega_{2}$ & $\omega_{3}$ \\
\hline 0.999001 & 0.0000547723 & $2.1781 \times 10^{-7}+i$ & $2.1781 \times 10^{-7}-i$ \\
1 & 0.0541419 & $0.0214162+1.00021 i$ & $0.0214162-1.00021 i$ \\
1.001 & 0.0756118 & $0.0379596+1.00065 i$ & $0.0379596-1.00065 i$ \\
1.002 & 0.0913455 & $0.0529917+1.00124 i$ & $0.0529917-1.00124 i$ \\
1.003 & 0.103949 & $0.0670958+1.00195 i$ & $0.0670958-1.00195 i$ \\
1.004 & 0.114444 & $0.080533+1.00276 i$ & $0.080533-1.00276 i$ \\
1.009 & 0.148257 & $0.141477+1.00796 i$ & $0.141477-1.00796 i$ \\
1.01 & 0.152474 & $0.152799+1.00919 i$ & $0.152799-1.00919 i$ \\
1.0111 & 0.156379 & $0.165005+1.01059 i$ & $0.165005-1.01059 i$ \\
\hline
\end{tabular}

TABLE 6: The characteristic roots $\lambda_{1,2}= \pm \omega_{1}, \lambda_{3,4}= \pm \omega_{2}, \lambda_{5,6}= \pm \omega_{3}$ for $v=0.01$ and $D=0.001$.

\begin{tabular}{lccc}
\hline$\kappa$ & $\omega_{1}$ & $\omega_{2}$ & $\omega_{3}$ \\
\hline 0.999001 & $0.0336136+0.980468 i$ & $0.000207226-0.0789312 i$ & $0.0322915-1.02011 i$ \\
1 & $0.0187794+0.989012 i$ & $0.000045675-0.0553234 i$ & $0.0183652-1.01118 i$ \\
1.001 & $0.0000595752+0.999965 i$ & $1.42435 \times 10^{-11}-0.00173207 i$ & $0.0000595711-1.00003 i$ \\
1.002 & 0.0540899 & $0.0213661+1.00021 i$ & $0.0213661-1.00021 i$ \\
1.003 & 0.0755428 & $0.0378711+1.00065 i$ & $0.0378711-1.00065 i$ \\
1.004 & 0.0912669 & $0.0528684+1.00123 i$ & $0.0528684-1.00123 i$ \\
1.009 & 0.137552 & $0.117813+1.00566 i$ & $0.117813-1.00566 i$ \\
1.01 & 0.14327 & $0.129617+1.00677 i$ & $0.129617-1.00677 i$ \\
1.0111 & 0.148654 & $0.142293+1.00805 i$ & $0.142293-1.00805 i$ \\
\hline
\end{tabular}

TABLE 7: The characteristic roots $\lambda_{1,2}= \pm \omega_{1}, \lambda_{3,4}= \pm \omega_{2}, \lambda_{5,6}= \pm \omega_{3}$ for $v=0.01$ and $D=1372.4$.

\begin{tabular}{lccc}
\hline$\kappa$ & $\omega_{1}$ & $\omega_{2}$ & $\omega_{3}$ \\
\hline 0.999001 & $2426.1+1401.08 i$ & $1400.71-2426.74 i$ & $0.962167-1.66652 i$ \\
1 & $2427.92+1402.13 i$ & $1401.76-2428.56 i$ & $0.962327-1.6668 i$ \\
1.001 & $2429.74+1403.18 i$ & $1402.81-2430.38 i$ & $0.962488-1.66708 i$ \\
1.002 & $2431.56+1404.23 i$ & $1403.86-2432.2 i$ & $0.962648-1.66735 i$ \\
1.003 & $2433.38+1405.28 i$ & $1404.91-2434.02 i$ & $0.962808-1.66763 i$ \\
1.004 & $2435.2+1406.33 i$ & $1405.96-2435.84 i$ & $0.962967-1.66791 i$ \\
1.009 & $2444.29+1411.58 i$ & $1411.21-2444.93 i$ & $0.963764-1.66929 i$ \\
1.01 & $2446.1+1412.63 i$ & $1412.26-2446.74 i$ & $0.963923-1.66956 i$ \\
1.0111 & $2448.1+1413.78 i$ & $1413.41-2448.74 i$ & $0.964098-1.66987 i$ \\
\hline
\end{tabular}

a rigid spherical shell filled with a fluid of constant density and volume and containing the test particle, while in the present study, we have assumed that the first primary is a fluid in the shape of a sphere with nonisotropic mass and density variation, given that, the test particle is contained in the fluid. The second primary and the test particle both have their masses varying arbitrarily with time at the same rate as the first primary.

In the previous study, the autonomized dynamical system with constant coefficients is gotten, only when the shell is empty or when the densities of the medium and the test particle are equal, while in the present study, such limitation do not arise. In the present study, we found two collinear equilibrium points on the line joining the centers of the fluid and the second primary with one at the center of the fluid and the other away from it. Further, circular equilibrium points exist on the $\xi \eta$-plane and pairs of out-of-plane points which depend on the arbitrary constant $\kappa \in(0, \infty)$, and density and mass parameters are found on $\xi \zeta$-plane. However, in the previous study, there is only one collinear equilibrium point located at the center of the rigid shell and a pair of the out-of plane equilibrium points which exist only for $\kappa>1$.

The linear stability analysis however turns out to be same as the equilibrium points on the line collinear with the centers of the primary of the autonomized system which are conditionally stable; while the equilibrium points on the $\xi \zeta$ plane and the circular points are unstable.

The result of our research work can be summarized as follows. The restricted problem under the framework of the Robe's [1] problem with three variable mass bodies, which vary arbitrarily with time at the same rate, has the equilibrium points which; are, the points near the center of the fluid, points on the circle (circular points), and pairs of out-ofplane points. These equilibrium points are analogous to the 
problem studied by Hallan and Rana [3] and their stability results are similar, though we have assumed that the first primary is a spherical fluid and that the masses of the test particle and primaries vary with time. The idea of the submarine's mass changing with time may perhaps seem impossible because it is a rigid body. However, from Archimedes' principle, a submarine floating or submerging depends on buoyancy which is controlled by the ballast tanks found between the submarine's inner and outer hulls. Submarine resting on the water surface has positive buoyancy which means it is less dense than water. When this happens, the ballast tanks are empty. For it to submerge, vents on top of the tanks are opened and water floods in thereby making the submarine denser than the sea water. In this case, the submarine has negative buoyancy. For it to float again, the water in the tanks will be forced out and the submarine becomes less dense than the water and eventually floats. In this study, the buoyancy force depends on the mass of the submarine $m_{3}$, the densities of the submarine, and the medium, as well as the volume of the submarine. And so by the simple relation, connecting mass, density, and volume, we understand that, when water is allowed into the ballast, as the mass of the submarine is changing so is the density until it becomes denser than the medium and sinks. When water is let out of the tanks, the mass and density of the submarine reduce and as a matter of fact, it will float when the medium is denser. Neutral buoyancy is attained when the weight of the submarine equals the amount of water it displaces. The submarine will neither rise nor sink in this state. Hence, with changing mass comes a changing density while the volume remains a constant.

This study may be useful in the investigations of the dynamic problem of Earth-size planets covered completely by a water envelope (water planets), and also the study of the small oscillation of the Earth's inner core taking into account the Moon's attraction during the course of evolution. The problem discussed in this paper is highly idealized and therefore calls for more research.

\section{References}

[1] H. A. G. Robe, "A new kind of 3-body problem," Celestial Mechanics and Dynamical Astronomy, vol. 16, no. 3, pp. 343-351, 1977.

[2] A. R. Plastino and A. Plastino, "Robe's restricted three-body problem revisited," Celestial Mechanics and Dynamical Astronomy, vol. 61, pp. 197-206, 1995.

[3] P. P. Hallan and N. Rana, "The existence and stability of equilibrium points in the Robe's restricted three-body problem," Celestial Mechanics and Dynamical Astronomy, vol. 79, no. 2, pp. 145155, 2001.

[4] B. Kaur and R. Aggarwal, "Robe's problem: its extension to 2 + 2 bodies," Astrophysics and Space Science, vol. 339, no. 2, pp. 283-294, 2012.

[5] J. Singh and L. M. Hafsah, "Robe's circular restricted three-body problem under oblate and triaxial primaries," Earth, Moon, and Planets, vol. 109, pp. 1-11, 2012.

[6] M. Dufour, Comptes Rendus Hebdomadairesde l'Accademie de Sciences, pp. 840-842, 1886.

[7] H. Gylden, "Die Bahnbewegungen in einem Systeme von zwei Körpern in dem Falle, dass die Massen Veränderungen unterworfen sind," Astronomische Nachrichten, vol. 109, no. 1-2, pp. 1-6, 1884.

[8] I. V. Meshcherskii, “Ein Specialfall des Gyldén'schen Problems (A. N. 2593)," Astronomische Nachrichten, vol. 132, no. 9, pp. 129-130, 1893.

[9] I. V. Meshcherskii, "Ueber die Integration der Bewegungsgleichungen im Probleme zweier Körper von veränderlicher Masse," Astronomische Nachrichten, vol. 159, pp. 229-242, 1902.

[10] J. Singh and O. Leke, "Stability of the photogravitational restricted three-body problem with variable masses," Astrophysics and Space Science, vol. 326, pp. 305-314, 2010.

[11] D. Veras, M. C. Wyatt, A. J. Mustill, A. Bonsor, and J. J. Eldridge, "The great escape: how exoplanets and smaller bodies desert dying stars," Monthly Notices of the Royal Astronomical Society, vol. 417, no. 3, pp. 2104-2123, 2011.

[12] J. Singh and O. Leke, "Existence and stability of equilibrium points in the Robe's restricted three-body problem with variable masses," International Journal of Astronomy and Astrophysics, vol. 3, pp. 113-122, 2013.

[13] E. P. Razbitnaya, "The problem of two bodies with variable masses-classification of different cases," Soviet Astronomy, vol. 29, pp. 684-687, 1985.

[14] A. A. Bekov, "Integrable cases of Hamilton-Jacobi equation and the restricted, rectilinear three-body problem with variable masses," Akademiya Nauk Kazakhskoi SSR, Trudy Astrofizicheskogo Instituta, vol. 12, p. 47, 1987.

[15] A. A. Bekov, A. N. Beysekov, and L. T. Aldibaeva, "On the dynamics of non-stationary binary stellar systems with nonisotropic mass flow," Astronomical \& Astrophysical Transactions, vol. 24, pp. 311-316, 2005.

[16] P. S. Letelier and T. A. da Silva, "Solutions to the restricted three-body problem with variable mass," Astrophysics and Space Science, vol. 332, no. 2, pp. 325-329, 2011.

[17] B. E. Gelf'gat, Modern Problems of Celestial Mechanics and Astrodynamics, Nauka, Moscow, Russia, 1973.

[18] A. A. Bekov, "Libration points of the restricted problem of three bodies of variable mass," Soviet Astronomy, vol. 32, no. 1, pp. 106-107, 1988.

[19] M. L. Krasnov, A. I. Kiselyov, and G. I. Makarenko, A Book of Problems in Ordinary Differential Equations, MIR, Moscow, Russia, 1983. 

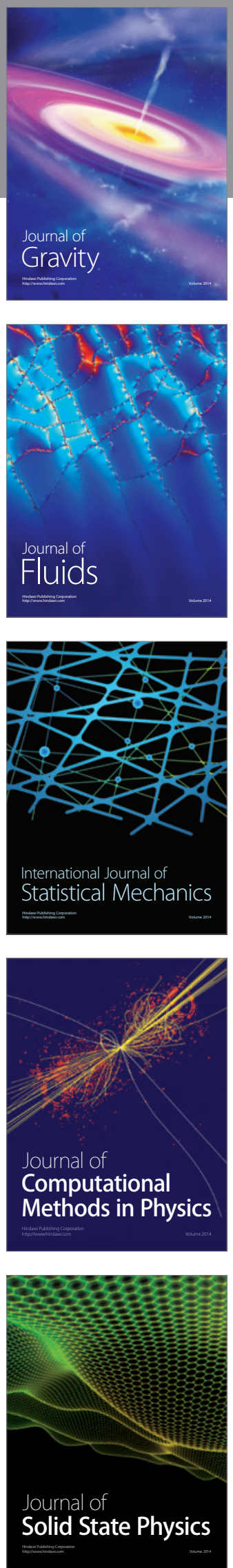

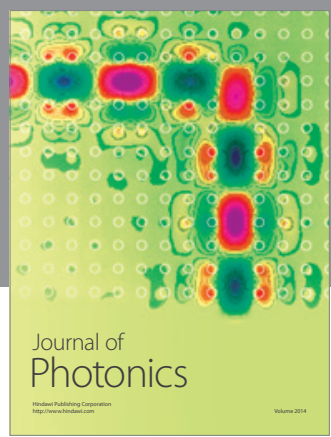

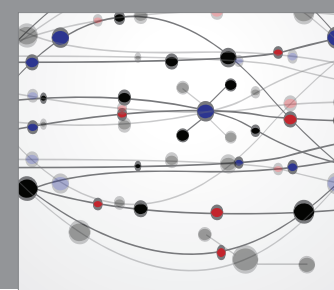

The Scientific World Journal

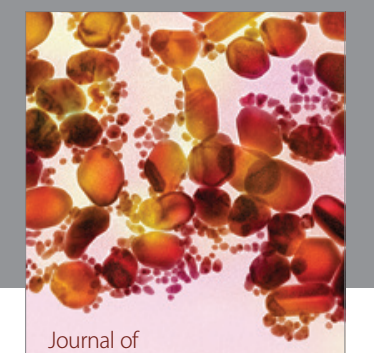

Soft Matter
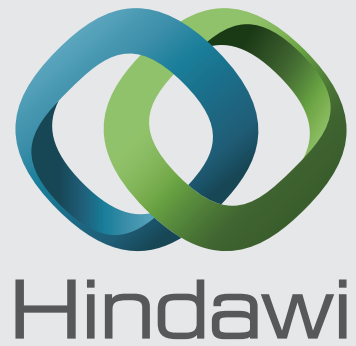

Submit your manuscripts at

http://www.hindawi.com
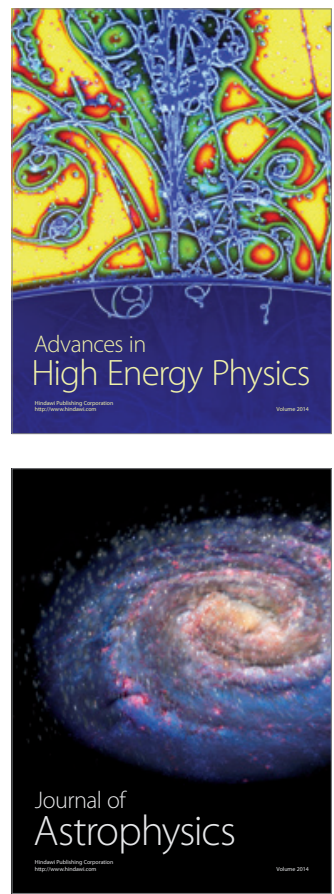
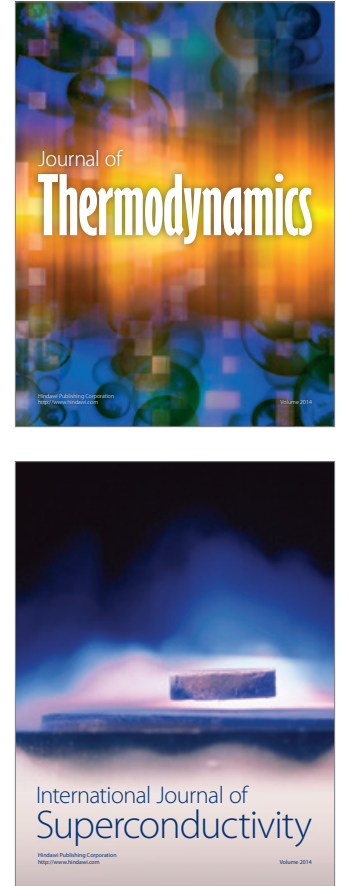
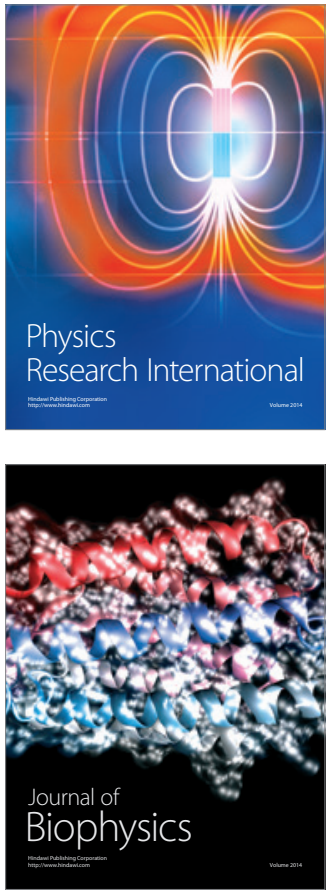
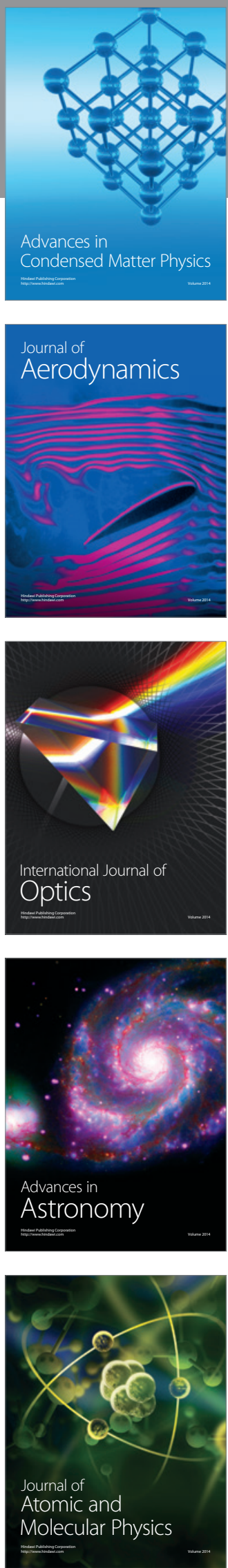